\title{
EDITORIAL
}

\section{The new hospital epidemiology}

Formerly, to demonstrate that epidemic spread of a particular microorganism had occurred in a hospital, it was deemed sufficient to type the infecting organism using phage, bacteriocin or serological methods. Although strictly such methods only establish differences between isolates, it was generally assumed that isolation of two or more staphylococci of the same phage type and antibiotic sensitivity pattern was evidence of the spread of a single strain. Although staphylococci and other epidemic organisms such as pseudomonas or klebsiclla clearly did acquire resistance to antibiotics from somewhere, this was rarely, if ever, recorded as occurring in the hospital environment, though this is the most likely locale due to the extensive use of antibioties. Noble et al. (1964) emphasized this when, in a study of 582 changes in nasal staphylococci amongst 2642 hospital patients, they wrote 'No clear evidence of a change in antibiotic sensitivity unaccompanied by a change in phage pattern was seen.'

In some current contexts, for example an outbreak of food poisoning or limited spread of some relatively obscure organism, it remains sufficient to establish that a particular species or biotype of organism is involved. Modern techniques for the study of DNA, however, reveal that the spread of genetic material may occur, independently of the spread of the host organisms, thus opening a new chapter in hospital epidemiology.

The ability of both Gram-negative and Gram-positive bacteria to accept and/or donate genetic material from other members of the same species, from other species of the same genus, or even from apparently unrelated microorganisms is now well established. In the laboratory, transfer of resistance genes can be accomplished by transduction in which phage is the vehicle, by transformation in which naked DNA enters the recipient or by conjugation in which some form of direct physical contact is made between the donor and recipient.

Until recently evidence for transmission of resistance plasmids rested on the demonstration that non-chromosomal DNA was associated with the antibiotic resistance pattern of the specific organism. The approximate molecular weight of the plasmid could be determined by centrifugation in a density gradient, by electrophoresis in an agarose gel or by measurement in electron micrographs. When the resistance was eliminated from the cells by growth in ethidium bromide, sodium dodecyl sulphate or at elevated temperature, the plasmid DNA was no longer demonstrable and, more importantly, when the resistance was transferred to another cell, the associated plasmid appeared in the newly resistant strain.

As genetic studies became more advanced however, it became evident that genes could be transferred in the absence of demonstrable plasmid DNA; lack of a newly transferred plasmid was no longer evidence of lack of transfer of genes. It was found 
that, especially amongst plasmids of Gram-negative bacilli, lengths of DNA could become inserted into the chromosome or into pre-existing plasmids, creating a plasmid with a slightly greater molecular weight; such DNA fragments are known as transposons. Recognition of transfer then became a question of recognition of specific segments of DNA. Two methods are available for accomplishing this in practice; one is the use of restriction endonuclease cleavage and the other DNA:DNA hybridization. Restriction endonucleases are enzymes that each recognize a different short sequence of DNA bases and sever the DNA at a point in that sequence. Thus plasmid DNA isolated by centrifugation is exposed to an endonuclease and the number and size of DNA fragments created examined by agarose gel electrophoresis. The similarity and presumptive identity of the samples can be established by cleaving aliquots of DNA with several different endonucleases. If plasmids from separate sources have several restriction sites and DNA fragments in common, they are assumed to have base sequences, i.e. genes, in common. In DNA:DNA hybridization, single strand radio-labelled DNA is obtained from one source and single strand DNA from a second source allowed to reassociate or hybridize with it and the percentage reassociation measured. This technique measures base sequence homology.

It is, then, possible to identify and compare specific segments of DNA from different sources and to establish that gene transfer has occurred even when the index DNA has been incorporated in pre-existing DNA. The scope which these techniques offer to hospital epidemiology is indicated by the following selection of publications.

As has generally proved true in microbial genetics, the best evidence is found amongst the Gram-negative bacilli. Thus Elwell, Inamine \& Minshew (1978), in a study of infection in a burns unit in which five different tobramycin resistant Gram negative bacilli had been isolated, were able, by endonuclease cleavage and DNA:DNA reassociation, to demonstrate the presence of a common plasmid mediating this resistance in Klebsiella pneumoniae and Enterobacter cloacae.

O'Brien et al. (1980) reported the spread of a plasmid mediating gentamicin resistance which appeared initially in a strain of $K$. pneumoniae. This organism disappeared from the hospital, but the same plasmid as determined by endonuclease cleavage, was recovered from isolates of Serratia marcescens, Enterobacter aerogenes, Escherichia coli, Citrobacter freundii and Proteus morganii over a period of about 18 months. By contrast in the study reported by Markowitz et al. (1980) only $K$. pneumoniae was involved, though several serotypes were implicated. A nursery for newborns was the venue for this epidemic which was due to multi-resistant organisms; a common plasmid was isolated from the various strains and identified by endonuclease cleavage. Experimentally the plasmid was shown to code for the appropriate antibiotic resistances and for self transfer. It is presumed that intestinal carriage amongst the patients and dissemination on the hands of staff maintained the epidemic spread. On a larger scale, O'Brien et al.(1982) demonstrated the similarity or identity of plasmids isolated from Salmonella species found in cattle and in humans over large areas of the United States. One serotype-plasmid combination was found to be endemic in cattle in 20 States and was also isolated from 26 human patients in two States. There was little or no evidence, other than that afforded by endonuclease cleavage of the plasmid DNA, to indicate that these 
persons' illnesses were in any way connected. The authors emphasize the implication that resistance plasmids are extensively shared by bacteria of apparently separate human and animal origin.

Spread of a suitable plasmid or transposon does not inevitably occur however. A $K$. aerogenes strain, followed by Datta et al. (1979) for a period of three months during a hospital outbreak of infection, carried a multi-resistance plasmid that was also found in $C$. koseri and Esch. coli isolated from the index patient but not from elsewhere. The plasmid incorporated a transposon for trimethoprim resistance but on this occasion it was not observed to spread.

It is usually assumed that the gut is the ecological niche in which gene transfer occurs between Gram-negative bacilli. However, Schaberg, Highsmith \& Wachsmuth (1977) reported that plasmid transfer may occur in the urinary catheter collection bag. Serratia do not often colonize the gut, so identification of an alternative habitat in which transfer occurs easily may be important. Others have demonstrated the ease with which plasmids coding for resistance to as many as nine antibiotics can be transferred from S. marcescens to Esch. coli, and that the $E s c h$. coli transconjugants were further able to transfer the plasmid.

It is now well attested that the $\beta$-lactamase resistance genes found in Neisseria gonorrhoeae have base sequences in common with those found in Haemophilus influenzae or in some Enterobacteriaceae and that plasmids of differing molecular weights have some sequences in common (e.g. Dickgiesser, Bennett \& Richmond, 1982; $\mathrm{Ng}$ et al. 1982). Beta lactamase genes in Haem. ducreyi from world wide sources are similar and moreover have sequences in common with $\beta$-lactamase plasmids in $N$. gonorrhoeae (Brunton et al. 1982). The overall plasmid profiles of many $\beta$-lactamase producing $N$. gonorrhoeae are different; but, since cryptic plasmids are also present, Elwell et al. (1977) suggested that insertion of gene sequences into pre-existing plasmids may have occurred. They further speculate that some fragments of DNA could prove exceptionally mobile and, as the extrachromosomal gene pool increases, plasmids with wider abilities to infect diverse groups of organisms will arise and be maintained.

Amongst the Gram-positive bacteria, the best evidence is of spread of genes amongst the staphylococci. Jaffe et al. (1980) have reported that, during an outbreak of infection in a neonatal special care nursery, gentamicin resistant strains of Staphylococcus aureus and S. epidermidis could be isolated. Plasmids recovered from five $S$. aureus and two $S$. epidermidis isolates were of the same molecular weight and restriction endonuclease cleavage patterns. The probability of transfer was demonstrated by experimental transmission of plasmids between strains on human skin. Totten, Vidal \& Baldwin (1981) have shown by endonuclease fingerprinting that tetracycline resistance plasmids from wild strains of $S$. aureus and $S$. epidermidis are indistinguishable but report that plasmids bearing genes for $\beta$-lactamase production had considerable heterogeneity. Nevertheless, Cohen, Wong \& Falkow (1982) reported the identification of a plasmid mediating $\beta$-lactamase production and resistance to gentamicin in $S$. aureus and $S$. epidermidis isolates from an outbreak of infection in a hospital ward and Weinstein et al. (1982) have demonstrated homology between plasmids found in paired $S$. aureus and $S$. epidermidis isolates in four separate incidents.

O'Reilly et al. (1981) reported on the homogeneity of plasmids which specify the 
production of type II epidermolytic toxin in S. aureus strains of various phage types. In a group of 22 distinct strains which carried a plasmid mediating toxin production, restriction endonuclease Hind III generated 22 DNA fragments, 19 of which were found in all strains. This implies transmission of a single plasmid between toxin producing strains.

Heterogeneity of plasmids can also be established. Naidoo et al. (1983) have reported that several different gentamicin resistance plasmids were involved in a multi-phage type outbreak of staphylococcal infection in a dermatology unit. Schiller, Groman \& Coyle (1980) demonstrated heterogeneity between erythromycin resistance plasmids in Corynebacterium diphtheriae and those in cutaneous corynebacteria isolated during an epidemic of cutaneous diphtheria.

The spread of virus in hospital wards has also been studied using DNA fingerprinting techniques. Linnemann et al. (1978) reported the use of endonuclease restriction cleavage to determine that two newborn infants in one hospital ward were infected with herpes simplex virus from separate sources. Adams et al. (1981) later reported an outbreak of cross-infection due to herpes simplex virus type 1 , two separate clusters of cases occured, each due to a separate virus strain.

A common theme recurring throughout papers reporting the epidemiology of plasmid transmission is that of establishing the source of resistance genes. It is suggested that endonuclease finger-printing or DNA homology studies will allow us to determine whether a pool of resistance genes, probably in plasmid form, is being maintained in the hospital environment, and will enable us, at least in principle, to track down and eliminate or reduce that pool. Whether the techniques involved can yet be regarded as within reach of the ordinary pathology laboratory seems doubtful, but they already provide an insight into a fascinating new aspect of hospital epidemiology.

W. C. Noble Editor

\section{REFERENCES}

Adams, G., Stover, B. H., Keenlyside, R. A., Hooton, T. M., Bucknan, T. G., Roizman, B. \& Stewart, J. A. (1981). Nosocomial herpetic infections in a pediatric intensive care unit. American Journal of Epidemiology 113, 126-132.

Brunton, J., Meier, M., Ehrman, N., Maclazan, I., Slaney, L. \& Alnritton, W. L. (1982). Molecular epidemiology of beta-lactamase-specifying plasmids of Haemophilus ducreyi. Antimicrobial Agents and Chemotherapy 21, 857-863.

Conen, M. L., Wona, E. S. \& Falkow, S. (1982). Common R plasmids in Staphylococcus aureus and Staphylococcus epidermidis during a nosocomial Staphylococcus aureus outbreak. Antimicrobial Agents and Chemotherapy 31, 210-215.

Datta, N., Hughes, V. M., Nugent, M. E. \& Richakds, H. (1979). Plasmids and transposons and their stability and mutability in bacteria isolated during an outbreak of hospital infection. Plasmids 2, 182-196.

Dickgiesser, N., Bennet, P. M. \& Richmond, M. H. (1982). Penicillinase-producing Neisseria gonorrhoeae: a molecular comparison of $5 \cdot 3 \mathrm{~Kb}$ and $7 \cdot 4 \mathrm{~Kb}$ B lactamase plasmids. Jourmal of Bacteriology 151, 1171-1175.

Elwell, L. P., Inamine, J. M. \& Minsuew, B. H. (1978). Common plasmid specifying tobrnmycin resistance found in two enteric bacteria isolated from burn patients. Antimicrobial Agents and Chemotherapy 13, 312-317.

Elweld, L. P., Roberts, M., Maykr, L. W. \& Falkow, S. (1977). Plasmid-mediated beta lactamase production in Neisseria gonorrhoeae. Antimicrobial Agents and Chemotherapy 11. 528-533. 
Jaffe, H. W., Swefeney, H. M., Nathan, C., Weinstein, R. A., Kabins, S. A. \& Cohen, S. (1980). Identity and interspecific transfer of gentamicin-resistance plasmids in Staphylococcus aureus and Staphylococcus epidermidis. Journal of Infectious Diseases 141, 738-747.

Linnemann, C. C. Jr., Buchman, T. G., Light, I. J., Ballard, J. L. \& Roizman, B. (1978). Transmission of herpes simplex virus type 1 in a nursery for the newborn. Identification of viral isolates by D.N.A. 'fingerprinting'. Lancet i, 964-966.

Markowitz, S. M., Veasey, J. M. Jr., Mlacrina, F. L., Mayhall, C. G. \& Lamb, V. A. (1980). Sequential outbreaks of infection due to Klebsiella pneumoniae in a neonatal intensive care unit: implication of a conjugative R plasmid. Journal of Infectious Diseases 142, 106-112.

Naidoo, J., Noble, W. C., Weissmans, A. \& Dyke, K. G. H. (1983). Gentamicin-resistant staphylococci: genetics of an outbreak in a dermatology department. Journal of Hygiene 91, 7-16.

Na, W. W. S., Echeverria, P., Rockhill, R. \& Dvangmani, C. (1982). Antibiotic sensitivities of Neisseria gonorrhoeae in the Far East: comparison of plasmid species in isolates from six countries. Sexually Transmitted Diseases 9, 120-123.

Noble, W. C., Williams, R. E. O., Jevons, MI. P. \& Shooter, R. A. (1964). Some aspects of nasal carriage of staphylococci. Journal of Clinical Pathology 17, 79-83.

O'Brien, T. F., Hopkins, J. D., Gilleece, E. S., Mediros, A. A., Kent, R. L., Blackburn, B. O., Holmes, M. B., Reardon, J. P., Vergeront, J. Ml., Schell, W. L., ChristenSON, E., BissetT, MI. L. \& Monse, E. V. (1982). Mlolecular epidemiology of antibiotic resistance in salmonella from animals and human beings in the United States. New England Journal of Medicine 307, 1-6.

O’Brien, T. F., Ross, D. G., Guzman, M. A., Mledeiros, A. A., Hedges, R. W. \& Botstein, D. (1980). Dissemination of an antibiotic resistance plasmid in hospital patient flora. Antimicrobial Agents and Chemotherapy 17, 537-543.

O'Reilly, M., Dougan, G., Foster, T. J. \& Arnutinotr, J. P. (1981). Plasmids in epidermolytic strains of Staphylococcus aureus. Journal of General Microbiology, 124, 99-107.

Schabera, D. R., Highsmiti, A. K. \& Wachsmutu, I. K. (1977). Resistance plasmid transfer by Serratia marcescens in urine. Antimicrobial Agents and Chemotherapy 11, 449-450.

Schiller, J., Groman, N. \& Coyle, MI. (1980). Plasmids in Corynebacterium diphtheriae and diphtheroids mediating erythromycin resistance. Antimicrobial Agents and Chemotherapy 18. 814-821.

Totten, P., Vidal, L. \& Baldwin, J. N. (1981). Penicillin and tetracycline resistance plasmids in Staphylococcus epidermidis. Antimicrobial Agents and Chemotherapy 20, 359-365.

Weinstein, R. A., Kabins, S. A., Nathan, C., Sweeney, H. M., Jaffe, H. W. \& Cohen, S. (1982). Gentamicin-resistant staphylococci as hospital flora: epidemiology and resistance plasmids. Journal of Infectious Diseases 145, 374-382. 